\title{
TU/e EmonOWEN

\section{Kinetic state diagrams for a highly asymmetric block copolymer assembled in solution}

\section{Citation for published version (APA):}

Vena, M. P., de Moor, D., laniro, A., Tuinier, R., \& Patterson, J. P. (2021). Kinetic state diagrams for a highly asymmetric block copolymer assembled in solution. Soft Matter, 17(4), 1084-1090.

https://doi.org/10.1039/d0sm01596b

\section{Document license:}

TAVERNE

DOI:

10.1039/d0sm01596b

Document status and date:

Published: 28/01/2021

\section{Document Version:}

Publisher's PDF, also known as Version of Record (includes final page, issue and volume numbers)

\section{Please check the document version of this publication:}

- A submitted manuscript is the version of the article upon submission and before peer-review. There can be important differences between the submitted version and the official published version of record. People interested in the research are advised to contact the author for the final version of the publication, or visit the $\mathrm{DOI}$ to the publisher's website.

- The final author version and the galley proof are versions of the publication after peer review.

- The final published version features the final layout of the paper including the volume, issue and page numbers.

Link to publication

\section{General rights}

Copyright and moral rights for the publications made accessible in the public portal are retained by the authors and/or other copyright owners and it is a condition of accessing publications that users recognise and abide by the legal requirements associated with these rights.

- Users may download and print one copy of any publication from the public portal for the purpose of private study or research.

- You may not further distribute the material or use it for any profit-making activity or commercial gain

- You may freely distribute the URL identifying the publication in the public portal.

If the publication is distributed under the terms of Article 25fa of the Dutch Copyright Act, indicated by the "Taverne" license above, please follow below link for the End User Agreement:

www.tue.nl/taverne

Take down policy

If you believe that this document breaches copyright please contact us at:

openaccess@tue.nl

providing details and we will investigate your claim. 


\section{Soft Matter}

Check for updates

Cite this: Soft Matter, 2021. 17, 1084

Received 3rd September 2020, Accepted 21st November 2020 DOI: $10.1039 / d 0 s m 01596 b$

rsc.li/soft-matter-journal

\section{Kinetic state diagrams for a highly asymmetric block copolymer assembled in solution $\dagger$}

\author{
M. Paula Vena, ${ }^{a b}$ Demi de Moor, ${ }^{e}$ Alessandro laniro, (DD ${ }^{\text {abc }}$ Remco Tuinier (D) ab and \\ Joseph P. Patterson (iD *df
}

\begin{abstract}
Polymer self-assembly is used to form nanomaterials with a wide range of structures. While self-assembly of polymers in bulk has been thoroughly explored, the same process in solution remains widely used but partially unresolved, due to the formation of structures which are often kinetically trapped. In this paper we report kinetic state diagrams of polystyrene- $b$-poly(ethylene oxide) block copolymer in water by changing the solvent-switch assembly conditions. We study 36 different conditions for a single block copolymer, exploring three parameters: polymer concentration, temperature and rate addition of selective solvent. The data shows that polymer concentration plays an important role in determining which morphologies are accessible within a given set of experimental parameters and provides evidence that vesicles can evolve into particles with complex internal structures, supportive of recent mechanistic studies. Most importantly, the data shows a complex relationship between all parameters and the resulting kinetically trapped morphologies indicating that combined in situ and ex situ studies are required to gain a fundamental understanding of kinetically controlled block copolymer assembly processes.
\end{abstract}

\section{Introduction}

Block copolymers can self-assemble in solution to form nanostructures that find application in catalysis, ${ }^{1}$ drug delivery ${ }^{2}$ and as templates for the synthesis of inorganic nanomaterials., The performance of these materials is often related to their ability to encapsulate, transport and release small molecules, which is highly dependent on their morphology. Consequently, there has been significant research dedicated to understanding how to predict and control the morphology of block copolymer assemblies. ${ }^{5}$ The most important consideration when investigating a specific block copolymer is whether the self-assembled structures form under thermodynamic or kinetic control. ${ }^{6}$

\footnotetext{
${ }^{a}$ Laboratory of Physical Chemistry, Department of Chemical Engineering and Chemistry, Eindhoven University of Technology, P.O. Box 513, 5600 MB, Eindhoven, The Netherlands

${ }^{b}$ Institute for Complex Molecular Systems, Eindhoven University of Technology, P.O. Box 513, 5600 MB, Eindhoven, The Netherlands

${ }^{c}$ Adolphe Merkle Institute, University of Fribourg, Chemin des Verdiers 4, CH-1700, Fribourg, Switzerland

${ }^{d}$ Department of Chemistry, University of California Irvine, 1102 Natural Sciences II, Irvine, California 92697, USA. E-mail: patters3@uci.edu

${ }^{e}$ Center for Multiscale Electron Microscopy, Department of Chemical Engineering and Chemistry, Eindhoven University of Technology, P. O. Box 513, 5600 MB Eindhoven, The Netherlands

${ }^{f}$ Department of Materials Science and Engineering, University of California, Irvine, Irvine, CA 92697-2025, USA

$\dagger$ Electronic supplementary information (ESI) available. See DOI: 10.1039/ dosm01596b
}

For thermodynamically controlled processes the morphology is determined by the macromolecular structure, the solution composition and the temperature. ${ }^{7}$ There are several theoretical approaches ${ }^{8}$ which can accurately predict the morphology of thermodynamically controlled block copolymer assemblies including self-consistent field (SCF) theory ${ }^{9-13}$ and density functional theory (DFT). ${ }^{14-16}$ Monte Carlo (MC) and (coarse grained) molecular dynamics simulations $(\mathrm{MD})^{17-20}$ can be performed to verify the theoretical results, ${ }^{8}$ or study cases where theoretical approaches fail. Experimentally, thermodynamically controlled assemblies are typically investigated by synthesizing a library of block copolymers with different molecular weights and block ratios and assembling them at a fixed condition. For the experimental and theoretical approaches, the results are typically displayed as phase diagrams, which show how the assembled morphology varies with the molecular composition of the polymer, typically focused on varying the block ratios. ${ }^{5}$ However, the approaches which have been successful for thermodynamically controlled assemblies fail to explain why the morphology of block copolymer assemblies is often dependent on the self-assembly pathway. ${ }^{21-25}$ For kinetically controlled processes the morphology is determined by the macromolecular structure and composition and the assembly method. Consequently, the investigation of kinetically controlled processes requires fundamentally different approaches. The most common approaches are:

(i) to use an assembly method which provides access to low free energy structures and then investigate the assembly as a 
function of macromolecular structure..$^{9,24,26,27}$ The results can then be largely interpreted from a thermodynamics perspective.

(ii) select a single macromolecular structure and investigate the assembly as a function of the assembly method. ${ }^{27-29}$ The results for these experiments must be interpreted from a mechanistic perspective considering the thermodynamics and kinetic of how the assemblies evolve in solution. Consequently, it is common to study the self-assembly mechanisms using in situ or ex situ analytical approaches that can resolve morphology evolution during self-assembly. ${ }^{30-33}$

While in situ and time resolved ex situ studies provide great insight into the mechanism of self-assembly, they are typically only applied to a small number of assembly pathways, as their application is time-consuming and can require high-end instrumentation. Here we investigate the assembly of a kinetically controlled block copolymer using a kinetic state diagram approach. The state diagrams show, for a single macromolecular structure, how the morphology varies as a function of the self-assembly conditions. As a model system we selected to study the self-assembly of a polystyrene- $b$-poly(ethylene oxide) block copolymer $\left(\mathrm{PS}_{250}-b-\mathrm{PEO}_{45}\right)$ using a tetrahydrofuran (THF) to water solvent-switch procedure. This is an ideal model system as polystyrene is well known to form kinetically trapped assemblies in water and crew-cut block copolymers are known to form a wide range of morphologies. ${ }^{31,34-38}$ The kinetic state diagrams are constructed by variation of the starting polymer concentration $\left(0.1,1.0\right.$ and $\left.10 \mathrm{mg} \mathrm{mL}^{-1}\right)$, the rate of water addition $\left(1 \mathrm{~mL} \mathrm{~min}^{-1}, 1 \mathrm{~mL} \mathrm{~h}^{-1}\right.$ or $\left.1 \mathrm{~mL} \mathrm{~d}^{-1}\right)$, and the solution temperature $\left(4,20,40,60{ }^{\circ} \mathrm{C}\right)$. The parameters were selected to span the range of commonly used assembly conditions. Each specific assembly condition is only performed once. This is a deliberate choice to use the experimental bandwidth to maximize the explored state space. The downside of this approach is that we do not obtain information on how reproducible each individual condition is; however, it does allow us to draw robust conclusions by comparing the result of multiple experiments where a single parameter is fixed. The purpose of constructing these kinetic state diagrams is three-fold: (1) assess the range of accessible morphologies for a given polymer within a given set of experimental conditions, (2) to determine how the selfassembly parameters influence the morphology and (3) provide insight into self-assembly mechanisms.

\section{Experimental}

$\mathrm{PS}_{250}-b-\mathrm{PEO}_{45}\left(M_{\mathrm{nNMR}}=28000 \mathrm{~g} \mathrm{~mol}^{-1}, D=1.17\right)$ was synthesized using atom transfer polymerization procedure. ${ }^{39}$ The assemblies were performed as follows: a $10 \mathrm{mg} \mathrm{mL}^{-1}$ stock solution of polymer in THF was prepared and aliquots were further diluted with THF to a final volume of $1 \mathrm{~mL}$ to reach the specific desired concentration $\left(0.1,1.0\right.$ or $\left.10 \mathrm{mg} \mathrm{mL}^{-1}\right)$ for each self-assembly experiment. The polymer solutions were stirred at a specific temperature $\left(4,20,40,60^{\circ} \mathrm{C}\right)$ and at fixed stirring rate (500 rpm) using a magnetic stirrer bar. Water was added with a

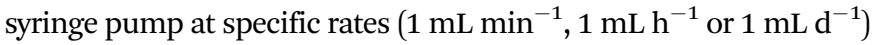

to achieve a solution composition of $1: 1 \mathrm{v} / \mathrm{v}$ THF $: \mathrm{H}_{2} \mathrm{O}$. The THF $: \mathrm{H}_{2} \mathrm{O}$ dispersions were dialyzed against water at room temperature to remove the THF. All other parameters which can influence the self-assembly were fixed (i.e. vial size, stirring bar size, stirring rate, syringe and needle size).

The dispersions were characterized using a TFS Tecnai Sphera at $200 \mathrm{kV}$. Dry state transmission electron microscopy is suitable for this study as it has previously been established that PS based block copolymer assemblies in water are stable to dehydration, ${ }^{38,40-43}$ including direct comparison of PS based assemblies using dry state and cryogenic TEM. ${ }^{43}$ In addition we performed cryo-TEM on selected samples to further confirm the stability of the assemblies to the dry state TEM preparation (Fig. S4, ESI $\dagger$ ). The dispersions were stored at room temperature and selected samples were characterized again after 1.5 years (Fig. S5, ESI $\dagger$ ). No changes were observed after 1.5 years of storage. Details on polymer synthesis, self-assembly experiments, characterization methods and morphology classification are given in the ESI. $\dagger$

\section{Results and discussion}

Self-assembly experiments were performed using a solventswitch procedure. During a solvent-switch procedure, polymers assemble as a consequence of an evolving energy landscape. ${ }^{44}$ In the pure common solvent (in this case THF), the lowest free energy state is the dissolved polymer and all the assembled states exist at a higher free energy. As the selective solvent (in this case water) is added, the energy landscape evolves such that the free energy of the dissolved state increases and the free energy of the assembled states decreases. ${ }^{5,44}$ Self-assembly occurs when the free energy of the dissolved polymer is higher than the free energy of an assembled state. However, multiple different assembled states can be at a lower free energy than the dissolved state. ${ }^{23}$ The self-assembly mechanism is dependent on the relative energies of each self-assembled state and the energy barriers between different states. The energy landscape evolves with each addition of the selective solvent. The morphology will continue to evolve if the energy barriers between states are comparable with the thermal energy. The morphology gets trapped in a meta-stable state when the energy barriers become significantly greater than the thermal energy. ${ }^{45}$ Variation in the polymer concentration provides access to different evolving energy landscapes. Variation in temperature affects both the evolving energy landscape and the available thermal energy. Variation in the addition rate of the selective solvent changes the kinetics with which the landscape evolves. Although not studied here, variation in the common solvent will also provide access to different evolving energy landscapes. ${ }^{27,28}$

In this study the assemblies were prepared via solventswitch, while three parameters were varied: initial polymer concentration, temperature and rate of water addition. This resulted in 36 aqueous dispersions of $\mathrm{PS}_{250}-b-\mathrm{PEO}_{45}$ assemblies that were characterized and classified by TEM. An initial screening by TEM revealed that $\mathrm{PS}_{250}-b-\mathrm{PEO}_{45}$ can form at least 


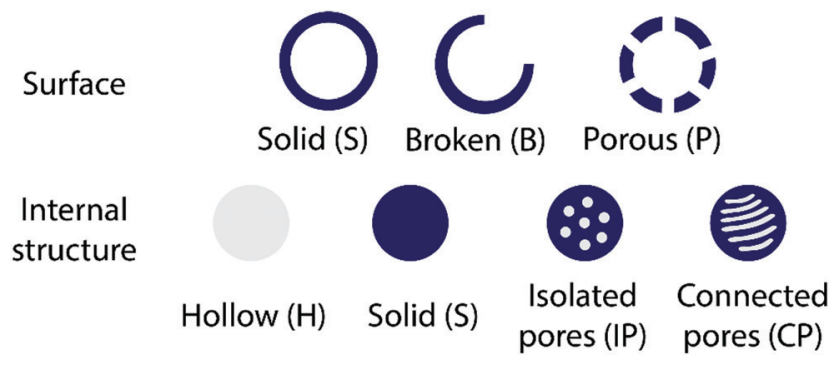

Fig. 1 Schematic representation of the different types of surface and internal structures observed for the self-assembly of $\mathrm{PS}_{250}-b-\mathrm{PEO}_{45}$. Top row: The surface can be classified as solid (S), broken (B) and porous (P). Low row: The internal structure can be classified as hollow $(\mathrm{H})$, solid (S), presents isolated pores (IP) or presents connected pores (CP).

nine different morphologies. These included large compound micelles, vesicles, nano-bowls, bicontinuous spheres and hexosomes that are common to block copolymer assemblies in water. ${ }^{5}$ Most morphologies were spherical and could be categorized by their surface and internal structures, as shown in Fig. 1. The surface structures were observed to be solid (S), broken (B) or porous (P) and the internal structures were observed to be hollow $(\mathrm{H})$, solid (S), contain isolated pores (IP) or contain connected pores (CP). An example of each morphology and a description of how each morphology was assigned is provided in the ESI. $\dagger$

This results in a matrix with 12 possible morphologies based on the combination of the different surfaces and internal structures (Fig. 2). A vesicle structure is categorized as having a solid surface and a hollow internal structure. A bicontinuous particle is categorized as having a porous surface and an internal structure with connected pores. A large compound micelle is categorized as having a solid surface and a solid internal structure. The purpose of this categorization is to show that a variety of nanocontainers with different encapsulation properties can be formed from a single block copolymer. ${ }^{46}$ Within the 36 dispersions, 9 of the 12 possible surface-internal structure combinations were found (dashed red squares in Fig. 2). This indicates that the self-assembly pathway plays a significant role in the formation of both the surface and internal structures of block copolymer nanoparticles.

To determine how the self-assembly parameters influence the morphology, kinetic state diagrams were constructed as a function of starting concentration, solvent-switch rate, and temperature. For clarity these are displayed as three two-dimensional kinetic state diagrams, and for samples containing multiple morphologies only the dominant morphology is presented (Fig. 3). The dominant morphology is based on number of particles by inspection of an average of 100 particles in the TEM micrographs. Depending on the specific application, it could be more appropriate to classify dominant morphologies based on particle mass. A complete description of each sample can be found in the ESI, $\dagger$ where it can be seen that the majority of samples contained multiple morphologies (see Table S1, ESI $\dagger$ ). The kinetic state diagrams show that all parameters studied play a role in the determination of the final morphology. A qualitative analysis of the state diagrams shows that, unlike the phase diagrams which are used for thermodynamically controlled assemblies, there are no obvious trends in the relationship between the variable and the final morphology. This indicates that, for this specific polymer, there is a complex relationship between the self-assembly mechanism and any modification to the evolving energy landscape.

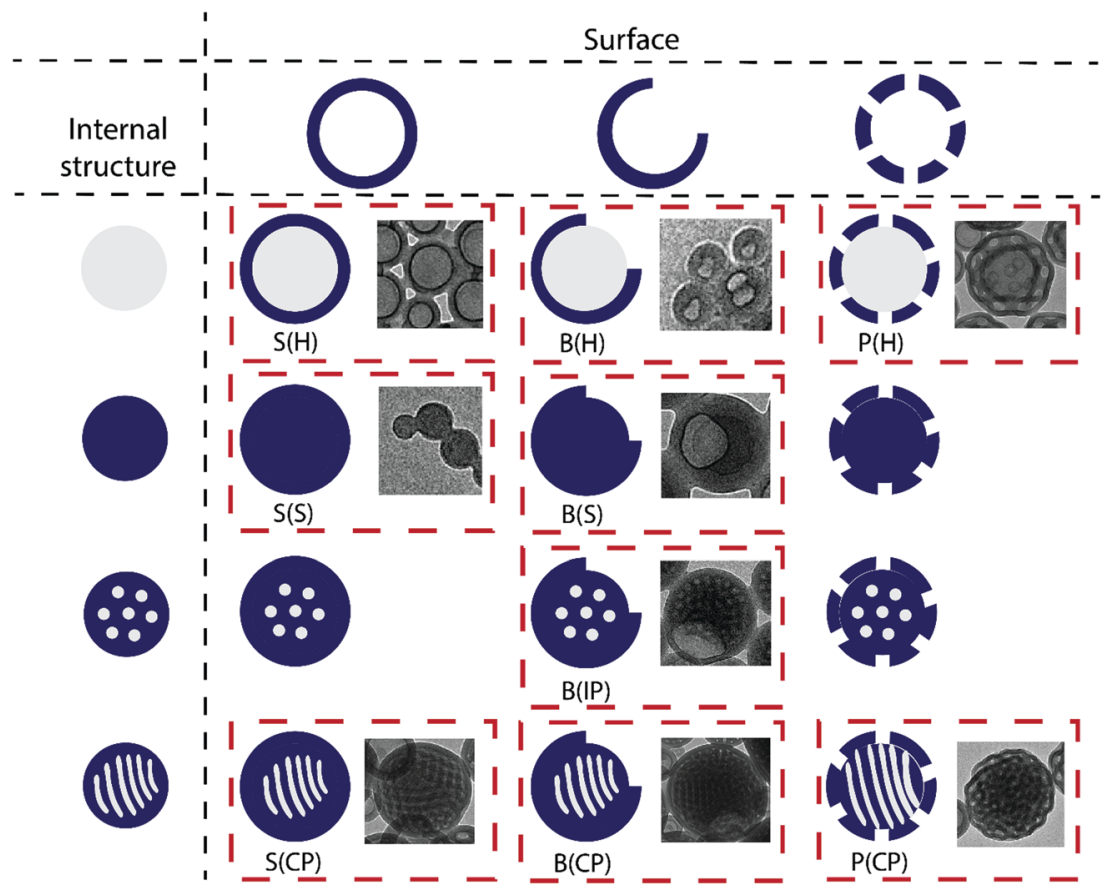

Fig. 2 Matrix of all possible combinations of surface and internal structures. The red dashed squares indicate the morphologies that have been found for $\mathrm{PS}_{250}-b-\mathrm{PEO}_{45}$ in this work. TEM micrographs are shown as examples for each morphology. 
a

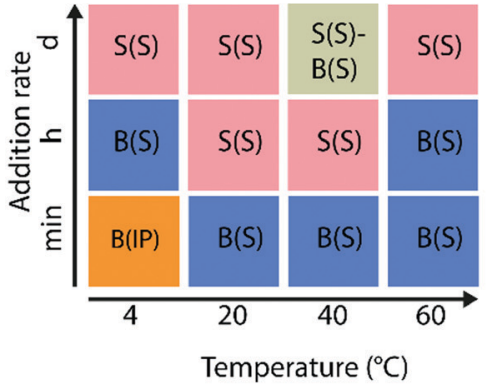

b Initial polymer concentration: $1 \mathrm{mg} / \mathrm{mL}$

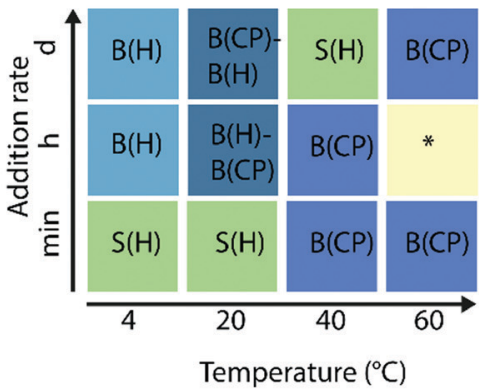

c

Initial polymer concentration: $10 \mathrm{mg} / \mathrm{mL}$

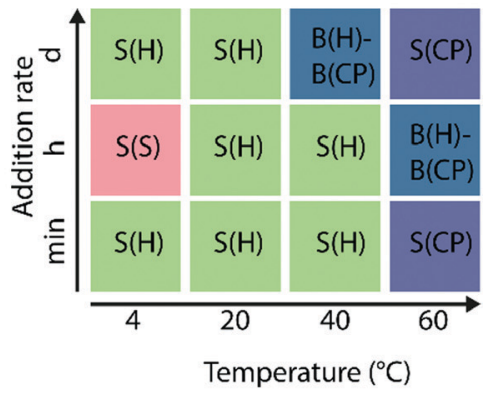

Fig. 3 State diagram obtained for $\mathrm{PS}_{250}-b-\mathrm{PEO}_{45}$ in function of temperature and water rate addition. For each condition only the predominant morphology in solution is showed. Each diagram was constructed at a fixed initial polymer concentration: (a) $0.1 \mathrm{mg} \mathrm{mL}^{-1}$, (b) $1 \mathrm{mg} \mathrm{mL}^{-1}$ and (c) $10 \mathrm{mg} \mathrm{mL}^{-1}$. See (Fig. 1 and 2) for an explanation of the symbols for each morphology.

However, the kinetic state diagrams show a strong relationship between the starting polymer concentration and the range of accessible morphologies. At a starting concentration of $10 \mathrm{mg} \mathrm{mL}{ }^{-1}$ vesicles $(\mathrm{S}(\mathrm{H}))$ were found to be the dominant morphology in 7 of the 10 samples, and found as a pure morphology in 2 samples. At a starting concentration of $0.1 \mathrm{mg} \mathrm{mL}^{-1}, 11$ out of the 12 samples formed with a solid core (either $\mathrm{B}(\mathrm{S})$ or $\mathrm{S}(\mathrm{S})$ ) and no samples formed vesicles as the dominant morphology. From a kinetic perspective, higher concentration self-assembly processes will have more collisions per unit time (a collision can involve individual polymer chains and/or aggregates of polymer chains). ${ }^{47,48}$ Consequently, it could be expected that high concentrations would lead to particles with higher aggregation numbers and larger sizes. Therefore, the results presented here, that vesicles are favored at higher concentration, might seem counterintuitive. However, higher polymer concentrations will result in the first assemblies being formed at lower water concentrations (i.e. earlier in the solvent switch process $)^{49}$ and we have previously shown that the time and to what degree a particle gets kinetically trapped during a solvent switch process is not only dependent on the solution composition but also on its configuration in the early stage of selfassembly. ${ }^{30}$ This again highlights that there is a complex relationship between the individual self-assembly parameters and the resulting kinetically trapped morphology. Size measurements performed for aggregates in each condition (without discriminating by morphology) shows that the average particle size increases with temperature, but there is only a weak trend with increasing polymer concentration (Fig. S12, ESI $\dagger$ ). It is important to note here that above $30{ }^{\circ} \mathrm{C}$, the solubility of the PEO chains in water decreases ${ }^{50}$ which will significantly affect the evolving energy landscape.

In 21 out of the 36 studied conditions, the assemblies present a broken surface. This so-called nano-bowl structure presents as a void that breaks the surface of the aggregate and has been hypothesized to form due to the collapse of the surface as THF is removed from the solution..$^{51}$ Interestingly, these structures are only found for 2 conditions at $10 \mathrm{mg} \mathrm{mL}^{-1}$, while they are present at almost all studied conditions at
0.1 and $1 \mathrm{mg} \mathrm{mL}^{-1}$ (Fig. 3). Nano-bowls with either a hollow internal structure $(\mathrm{B}(\mathrm{H})$ ) or with internal pores $(\mathrm{B}(\mathrm{IP})$ and $\mathrm{B}(\mathrm{CP})$ ) are observed when the concentration is increased, providing further evidence of the relationship between concentration and which morphologies are accessible.

Although it is known that polymer concentration can influence the morphology of kinetically trapped block copolymers, ${ }^{22,29,49}$ we believe that concentration is often overlooked when solvent switch protocols are designed. Solvent switch procedures are often formed in two stages, Stage 1 being the addition of the nonsolvent and Stage 2 the dialysis into a pure non-solvent. In Stage 1 there is a continuous decrease in polymer concentration whereas in stage two polymer concentration is fixed. Note: it is possible that concentration can change in stage 2 due to osmotic pressures difference resulting in the swelling of the dialysis bag, however this is typically not controllable. Consequently, designing a solvent switch procedure where self-assembly occurs at a specific concentration would require knowledge of the solution composition window where self-assembly is initiated and then becomes kinetically trapped. This window depends on the polymer composition, concentration and the specific solvents used. ${ }^{28}$

The state diagrams (see Table S1, ESI $\dagger$ ) also provide information about the mechanistic connection between certain morphologies. The solvent switch procedure traps meta-stable structures as they evolve. Consequently, morphologies that often appear together likely represent different stages in the formation mechanism. Therefore it is logical to create an image series that depicts a plausible mechanism based on the observation of many individual structures. ${ }^{52}$ However, it should be noted that in situ TEM studies have found that each individual assembled structure evolves down a unique pathway, ${ }^{30}$ and therefore this method can only be used to provide information on the general morphology evolution and does not provide a specific temporal series. The data presented in this work, indicates a mechanistic connection between vesicles $(\mathrm{S}(\mathrm{H})$ ) and particles with internal pores $(\mathrm{S}(\mathrm{CP})$ and $\mathrm{P}(\mathrm{CP})$ ) as they frequently appear together (Table S1, ESI $\dagger$ ). A size comparison of these structures formed under 5 different conditions at an initial polymer concentration of $10 \mathrm{mg} \mathrm{mL}{ }^{-1}$ reveals that in 

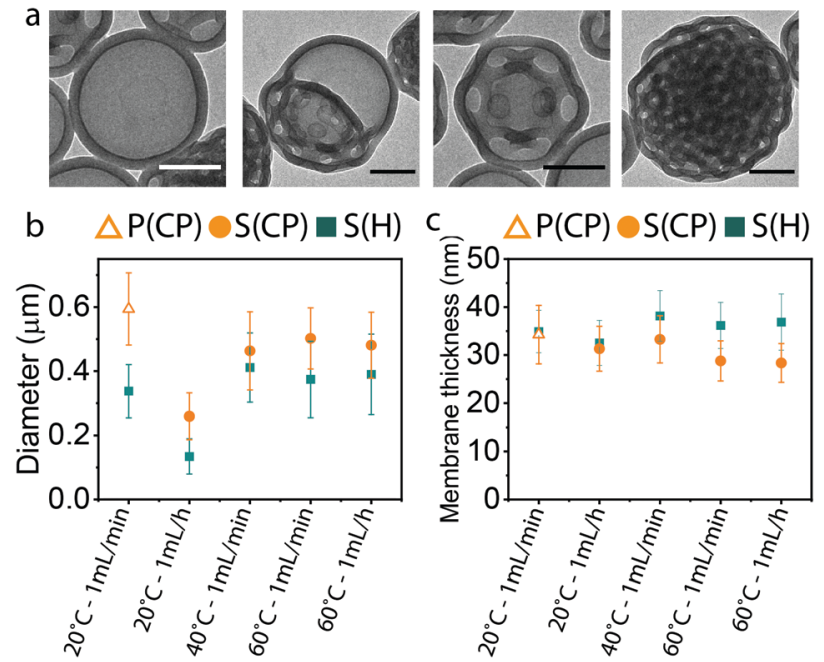

Fig. 4 (a) TEM characterization of the $\mathrm{PS}_{250}-b-\mathrm{PEO}_{45}$ assemblies (for initial polymer concentration $10 \mathrm{mg} \mathrm{mL}^{-1}$, temperature $20{ }^{\circ} \mathrm{C}$ and rate addition of $1 \mathrm{~mL} \mathrm{~min}^{-1}$ ). The selected images provide insight into a general morphology trend but do not represent a temporal series. The coexistence of $S(H), P(H)$ and $P(C P)$ indicates that these morphologies are mechanistically related. Scale bars: $200 \mathrm{~nm}$. (b) Particle size and (c) membrane thickness of coexisting morphologies $\mathrm{S}(\mathrm{H})(\square), \mathrm{S}(\mathrm{CP})(\mathrm{O})$ and $\mathrm{P}(\mathrm{CP})\left(\triangle\right.$ ) observed for $\mathrm{PS}_{250}-b-\mathrm{PEO}_{45}$ assemblies with initial concentration of $10 \mathrm{mg} \mathrm{mL}^{-1}$ at different temperatures and water rate addition.

each case the vesicles are significantly smaller than the particles with internal pores (Fig. 4b). This indicates that vesicles evolve into the particles with complex internal structures (Fig. 4a and Fig. S9, ESI $\dagger$ ) as it can be expected that the structures will generally increase in size as a mechanism progresses. At higher temperatures $\left(40{ }^{\circ} \mathrm{C}\right.$ and $\left.60{ }^{\circ} \mathrm{C}\right)$ the average membrane thickness of the vesicles $(\mathrm{S}(\mathrm{H}))$ is larger than for the structures with internal connected pores (Fig. 4c). This data is supportive of recent mechanistic studies. ${ }^{29,31,53}$ Lin et al. ${ }^{31}$ performed a timeresolved experiment taking aliquots during the self-assembly in DMF : $\mathrm{H}_{2} \mathrm{O}$ of two highly asymmetric block copolymers, $\mathrm{PS}_{236}-b$ $\mathrm{PEO}_{45}$ and $\mathrm{PS}_{334}-b-\mathrm{PEO}_{45}$, and observed that upon increasing the water content, the system evolves from vesicles to what they called large compound vesicles and sponge-like particles, classified as $\mathrm{S}(\mathrm{H}), \mathrm{P}(\mathrm{H})$ and $\mathrm{P}(\mathrm{CP})$, respectively, in our work. The sponge-like particles further evolved into cubosomes in the case of $\mathrm{PS}_{236}-b-\mathrm{PEO}_{45}$ and hexosomes for $\mathrm{PS}_{334}-b-\mathrm{PEO}_{45}$. For the purpose of this study we refer to both cubosomes and hexosomes as S(CP). The authors observed that the transition from vesicles to sponge-like aggregates and hexosomes involves an increase in particle diameter and decrease in membrane thickness, ${ }^{31}$ which coincides with the size trend we observe for the coexisting $\mathrm{S}(\mathrm{H})$ and $\mathrm{S}(\mathrm{CP})$ morphologies. Yu et al. ${ }^{29}$ studied the self-assembly of $\mathrm{PS}_{144}-b-\mathrm{PAA}_{22}$ in toluene:methanol mixtures at different polymer concentrations and solvents ratio. ${ }^{29}$ Instead of following a self-assembly approach, the polymer was added to the solvent mixture and aliquots were taken over 8 hours for characterization. The assemblies undergo a transformation from vesicles that fuse into lamellae and sponge-like particles to finally reorganize into structures with ordered porous network internal structures. Parent et al. ${ }^{53}$ combined in situ liquid-cell TEM observations of block copolymer micelles with molecular dynamics simulations to show that micellar and vesicle fusion leads to structures with more complex internal structures. Similar observations can be made for $\mathrm{B}(\mathrm{H})$ and $\mathrm{B}(\mathrm{CP})$ assemblies, that coexist in five different samples (Fig. S11, ESI $\dagger$ ). Due to the complex nature of the assembly process and the wide range of structures that can form, a complete interpretation of the data from the state diagram would require automated analysis and is beyond the scope of this report. However, we believe this approach would be highly valuable when combined with data from complex in situ measurements of polymer assembly mechanisms.

\section{Conclusions}

The self-assembly of $\mathrm{PS}_{250}-b$ - $\mathrm{PEO}_{45}$ block copolymers was studied using a kinetic state diagram approach. State diagrams were constructed by performing solvent-switch experiments and varying the evolving energy landscape. The parameters studied here were the starting polymer concentration, the temperature of the solution and the solvent-switch rate resulting in 36 unique self-assembly processes. An important design feature in the state diagrams is to maximize the explored state space by only performing each specific assembly process once. Although this decision reduces confidence in the conclusions drawn from any individual experiment, it greatly increases confidence in the conclusions drawn from the collective experiments. This is important because the purpose of the state diagram approach is to draw general conclusions about the effect of specific parameters. For example, we performed 12 experiments at each concentration to draw conclusions about the general effect of concentration.

Our collective experiments show that polymer concentration plays a significant role in determining the range of accessible morphologies, a parameter which is often overlooked when designing solvent-switch experiments. Our data supports previous evidence ${ }^{31}$ that vesicles can evolve into particles with complex internal structures that result in an increase in particle size but a decrease in membrane thickness. Perhaps the most important observation is that all experimental parameters can strongly influence the final morphology, and the relationship between the variable and the outcome is complex. This provides further evidence that improving our fundamental understanding of kinetically controlled block copolymer assembly will require detailed mechanistic studies that can probe morphology evolution as a consequence of the evolving energy landscape. In this effort we believe that the application of kinetic state diagrams is useful for (1) assessing the range of accessible morphologies, (2) determining which morphologies are accessible within a given region of the state space and (3) for providing a basic understanding of the mechanistic connection between different morphologies. Importantly, any mechanisms proposed using complex in situ data should be 
consistent with the results of obtained from the kinetic state experiments. One limitation of the kinetic state diagram approach is the time-consuming nature of the experiments. However, recent developments in the automation of selfassembly experiments and analysis by transmission electron microscopy ${ }^{54}$ provide exciting opportunities to apply this approach more broadly.

\section{Conflicts of interest}

There are no conflicts to declare.

\section{Acknowledgements}

M. P. Vena was supported financially by the EU H2020 Marie Skłodowska-Curie Action project MULTIMAT. The authors acknowledge Stefan Govers (TUe) for the GPC data.

\section{References}

1 Z. B. Shifrina, V. G. Matveeva and L. M. Bronstein, Chem. Rev., 2019, 120, 1350-1396.

2 H. Cabral, K. Miyata, K. Osada and K. Kataoka, Chem. Rev., 2018, 118, 6844-6892.

3 L. A. Connal, N. A. Lynd, M. J. Robb, K. A. See, S. G. Jang, J. M. Spruell and C. J. Hawker, Chem. Mater., 2012, 24, 4036-4042.

4 T. Smart, H. Lomas, M. Massignani, M. V. Flores-Merino, L. R. Perez and G. Battaglia, Nano Today, 2008, 3, 38-46.

5 Y. Mai and A. Eisenberg, Chem. Soc. Rev., 2012, 41, 5969-5985.

6 T. Nicolai, O. Colombani and C. Chassenieux, Soft Matter, 2010, 6, 3111-3118.

7 E. B. Zhulina, M. Adam, I. LaRue, S. S. Sheiko and M. Rubinstein, Macromolecules, 2005, 38, 5330-5351.

8 T. E. Gartner III and A. Jayaraman, Macromolecules, 2019, 52, 755-786.

9 A. Ianiro, J. P. Patterson, Á. González García, M. M. van Rijt, M. M. M. Hendrix, N. A. Sommerdijk, I. K. Voets, A. C. C. Esteves and R. Tuinier, J. Polym. Sci., Part B: Polym. Phys., 2018, 56, 330-339.

10 F. A. M. Leermakers, C. M. Wijmans and G. J. Fleer, Macromolecules, 1995, 28, 3434-3443.

11 F. Li, M. Schellekens, J. de Bont, R. Peters, A. Overbeek, F. A. M. Leermakers and R. Tuinier, Macromolecules, 2015, 48, 1194-1203.

12 F. S. Bates and G. H. Fredrickson, Annu. Rev. Phys. Chem., 1990, 41, 525-557.

13 G. Fredrickson, The equilibrium theory of inhomogeneous polymers, Oxford University Press on Demand, 2006.

14 J. Wu and Z. Li, Annu. Rev. Phys. Chem., 2007, 58, 85-112.

15 J. Xia, M. Sun, F. Qiu, H. Zhang and Y. Yang, Macromolecules, 2005, 38, 9324-9332.

16 W. E. McMullen and K. F. Freed, J. Chem. Phys., 1990, 92, 1413-1426.

17 D. J. Beltran-Villegas and A. Jayaraman, J. Chem. Eng. Data, 2018, 63, 2351-2367.
18 I. Lyubimov, D. J. Beltran-Villegas and A. Jayaraman, Macromolecules, 2017, 50, 7419-7431.

19 A. Milchev, A. Bhattacharya and K. Binder, Macromolecules, 2001, 34, 1881-1893.

20 G. Srinivas, D. E. Discher and M. L. Klein, Nat. Mater., 2004, 3, 638-644.

21 K. Yu, L. Zhang and A. Eisenberg, Langmuir, 1996, 12, 5980-5984.

22 L. Zhang and A. Eisenberg, Macromolecules, 1999, 32, 2239-2249.

23 H. Cui, Z. Chen, S. Zhong, K. L. Wooley and D. J. Pochan, Science, 2007, 317, 647-650.

24 S. Jain and F. S. Bates, Science, 2003, 300, 460-464.

25 S. Jain and F. S. Bates, Macromolecules, 2004, 37, 1511-1523.

26 Y.-Y. Won, A. K. Brannan, H. T. Davis and F. S. Bates, J. Phys. Chem. B, 2002, 106, 3354-3364.

27 S. A. Barnhill, N. C. Bell, J. P. Patterson, D. P. Olds and N. C. Gianneschi, Macromolecules, 2015, 48, 1152-1161.

28 P. Bhargava, J. X. Zheng, P. Li, R. P. Quirk, F. W. Harris and S. Z. Cheng, Macromolecules, 2006, 39, 4880-4888.

29 H. Yu, X. Qiu, S. P. Nunes and K.-V. Peinemann, Nat. Commun., 2014, 5, 1-10.

30 A. Ianiro, H. Wu, M. M. van Rijt, M. P. Vena, A. D. Keizer, A. C. C. Esteves, R. Tuinier, H. Friedrich, N. A. Sommerdijk and J. P. Patterson, Nat. Chem., 2019, 11, 320-328.

31 Z. Lin, S. Liu, W. Mao, H. Tian, N. Wang, N. Zhang, F. Tian, L. Han, X. Feng and Y. Mai, Angew. Chem., Int. Ed., 2017, 56, 7135-7140.

32 K. Sankhala, D. C. F. Wieland, J. Koll, M. Radjabian, C. Abetz and V. Abetz, Nanoscale, 2019, 11, 7634-7647.

33 E. G. Kelley, R. P. Murphy, J. E. Seppala, T. P. Smart, S. D. Hann, M. O. Sullivan and T. H. Epps, Nat. Commun., 2014, 5, 3599.

34 J. Zhao, W. Huang, P. Si, J. Ulstrup, F. Diao and J. Zhang, Macromol. Rapid Commun., 2018, 39, 1800125.

35 K. T. Kim, J. Zhu, S. A. Meeuwissen, J. J. L. M. Cornelissen, D. J. Pochan, R. J. Nolte and J. C. van Hest, J. Am. Chem. Soc., 2010, 132, 12522-12524.

36 K. Yu and A. Eisenberg, Macromolecules, 1996, 29, 6359-6361. 37 K. Yu and A. Eisenberg, Macromolecules, 1998, 31, 3509-3518. 38 A. Cho, Y. La, S. Jeoung, H. R. Moon, J.-H. Ryu, T. J. Shin and K. T. Kim, Macromolecules, 2017, 50, 3234-3243.

39 W. Tang, Y. Kwak, W. Braunecker, N. V. Tsarevsky, M. L. Coote and K. Matyjaszewski, J. Am. Chem. Soc., 2008, 130, 10702-10713.

40 J. P. Patterson, M. P. Robin, C. Chassenieux, O. Colombani and R. K. O'Reilly, Chem. Soc. Rev., 2014, 43, 2412-2425.

41 J. Xu, Y. Wu, K. Wang, L. Shen, X. Xie and J. Zhu, Soft Matter, 2016, 12, 3683-3687.

42 Z. Lin, S. Liu, W. Mao, H. Tian, N. Wang, N. Zhang, F. Tian, L. Han, X. Feng and Y. Mai, Angew. Chem., 2017, 129, 7241-7246.

43 J. P. Patterson, A. M. Sanchez, N. Petzetakis, T. P. Smart, I. I. I. T. H. Epps, I. Portman, N. R. Wilson and R. K. O'Reilly, Soft Matter, 2012, 8, 3322-3328.

44 E. Mattia and S. Otto, Nat. Nanotechnol., 2015, 10, 111-119. 45 V. J. Anderson and H. N. W. Lekkerkerker, Nature, 2002, 416, 811-815. 
46 J. Wang, J. Wan, N. Yang, Q. Li and D. Wang, Nat. Rev. Chem., 2020, 1-10.

47 B. K. Johnson and R. K. Prud'homme, Phys. Rev. Lett., 2003, 91, 118302.

48 J. Aubry, F. Ganachaud, J.-P. Cohen Addad and B. Cabane, Langmuir, 2009, 25, 1970-1979.

49 A. Choucair and A. Eisenberg, Eur. Phys. J. E, 2003, 10, 37-44.

50 W. F. Polik and W. Burchard, Macromolecules, 1983, 16, 978-982.
51 I. C. Riegel, A. Eisenberg, C. L. Petzhold and D. Samios, Langmuir, 2002, 18, 3358-3363.

52 D. E. Discher and A. Eisenberg, Science, 2002, 297, 967-973. 53 L. R. Parent, E. Bakalis, A. Ramírez-Hernández, J. K. Kammeyer, C. Park, J. De Pablo, F. Zerbetto, J. P. Patterson and N. C. Gianneschi, J. Am. Chem. Soc., 2017, 139, 17140-17151.

54 M. A. Touve, D. B. Wright, C. Mu, H. Sun, C. Park and N. C. Gianneschi, Macromolecules, 2019, 52, 5529-5537. 